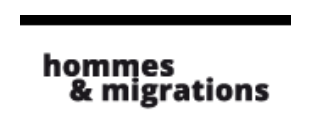

Hommes \& migrations

Revue française de référence sur les dynamiques

migratoires

1277 | 2009

France - Allemagne

\title{
"Des gens pas comme nous" les transmigrants turcs dans le documentaire allemand
}

\section{Barbara Laubenthal et Jörg-Uwe Nieland}

Traducteur : Françoise Toraille

\section{(2) OpenEdition}

\section{Journals}

Édition électronique

URL : http://journals.openedition.org/hommesmigrations/172

DOI : 10.4000/hommesmigrations. 172

ISSN : 2262-3353

Éditeur

Musée national de l'histoire de l'immigration

Édition imprimée

Date de publication : 1 janvier 2009

Pagination : 98-102

ISSN : 1142-852X

Référence électronique

Barbara Laubenthal et Jörg-Uwe Nieland, " "Des gens pas comme nous" les transmigrants turcs dans le documentaire allemand », Hommes \& migrations [En ligne], 1277 | 2009, mis en ligne le 29 mai 2013, consulté le 21 avril 2019. URL : http://journals.openedition.org/hommesmigrations/172 ; DOI :

10.4000/hommesmigrations. 172 


\section{"Des gens pas comme nous" les transmigrants turcs dans le documentaire allemand}

Par Barbara Laubenthal et Jörg-Uwe Nieland, université de la Ruhr, Bochum 
En 2005, 1764041 Turcs vivaient en Allemagne. Leur présence remonte à la période d'une politique de recrutement de travailleurs étrangers menée par la République fédérale dans les années 1950 et 1960. Cette immigration de maind'ceuvre a fait l'objet de nombreuses études sur l'intégration des migrants dans la société allemande. Au cours de la dernière décennie, les phénomènes migratoires dépassant de plus en plus les frontières des États, la recherche sur les migrants a élargi son terrain d'analyse en se penchant à la fois sur le pays d'accueil et sur celui d'origine. Les chercheurs ont créé le nouveau concept de transnationalisme pour décrire le processus permettant à certains immigrés de se construire et de maintenir des relations sociales d'enracinements multiples qui relient société d'origine et société de résidence. Cette orientation de la recherche a aussi élaboré la typologie de ces migrants d'un nouveau genre, les transmigrants, dont les champs sociaux font se croiser les frontières géographiques, culturelles et politiques (voir le tableau ci-dessous).

\section{Tableau : les types idéaux des migrants}

\begin{tabular}{|c|c|c|c|c|}
\hline & $\begin{array}{l}\text { Relation } \\
\text { à la région } \\
\text { d'origine }\end{array}$ & $\begin{array}{l}\text { Relation } \\
\text { à la région } \\
\text { d'arrivée }\end{array}$ & $\begin{array}{c}\text { Raisons } \\
\text { principales } \\
\text { de la migration }\end{array}$ & $\begin{array}{c}\text { Durée } \\
\text { envisagée } \\
\text { de la migration }\end{array}$ \\
\hline $\begin{array}{l}\text { Émigration / } \\
\text { Immigration }\end{array}$ & $\begin{array}{l}\text { Référence / } \\
\text { Adieu }\end{array}$ & $\begin{array}{l}\text { Intégration / } \\
\text { Nouvelle patrie }\end{array}$ & $\begin{array}{l}\text { Économiques / } \\
\text { Socio-culturelles }\end{array}$ & $\begin{array}{l}\text { Illimitée / } \\
\text { Long terme }\end{array}$ \\
\hline $\begin{array}{l}\text { Migration avec } \\
\text { perspective de retour }\end{array}$ & $\begin{array}{l}\text { Relation durable / } \\
\text { Préserver son identité }\end{array}$ & $\begin{array}{l}\text { Distance / } \\
\text { Pays d'accueil }\end{array}$ & $\begin{array}{l}\text { Économiques / } \\
\text { Politiques }\end{array}$ & $\begin{array}{l}\text { Limitée / } \\
\text { Court terme }\end{array}$ \\
\hline Transmigration & $\begin{array}{l}\text { Ambivalente / } \\
\text { Mêlée }\end{array}$ & $\begin{array}{l}\text { Ambivalente / } \\
\text { Mêlée }\end{array}$ & $\begin{array}{l}\text { Économiques / } \\
\text { D'organisation }\end{array}$ & $\begin{array}{l}\text { Inconnue / } \\
\text { Séquentielle }\end{array}$ \\
\hline
\end{tabular}

Source : Pries (2007a : 34)

Afin de cerner les caractéristiques des transmigrants l'étude d'Alejandro Portes ${ }^{(1)}$ trouve "un nombre croissant de personnes vivant une existence double : elles parlent deux langues, ont un foyer dans deux pays et construisent leur existence à travers des contacts réguliers et permanents par-delà les frontières nationales.” De même Ludger Pries ${ }^{(2)}$ qui a interviewé cette catégorie de migrants précise : "Ils ont évoqué les avantages et inconvénients de leur région d'arrivée, mais aussi de leur région de départ. Interrogés sur leurs projets d'existence à long terme, ils ont souvent indiqué n'en avoir que pour les deux ou trois années à venir, pour voir ensuite "quelles intentions Dieu a à mon égard". [...] Le transmigrant typique changeait cinq fois et plus de pays au cours de sa période de migration et de sa biographie de personne active." Le transmigrant garde donc une importante mobilité et franchit fréquemment des frontières nationales, tant sur le plan physique que mental. Ces changements de lieu géographique 
et mental sont parfaitement maîtrisés et suivent une forme de routine. Pour le transmigrant, "l'alternance entre lieux de vie dans différents pays devient normalite"”(3). Le transmigrant apparaît comme acteur d'un cosmopolitisme moderne.

\section{Les transmigrants précaires et le documentaire d'Aysun Bademsoy}

Le travail de conceptualisation mené jusqu'à présent pour définir le transmigrant est néanmoins incomplet. Il existe un groupe de migrants qui se distingue de façon significative des types idéaux décrits ci-dessus. On peut qualifier ce nouveau type de migrant de transmigrant précaire. Si les transmigrants passent d'un lieu à l'autre sans difficulté, cet autre type de transmigrant vit sur les plans psychique et émotionnel dans un no man's land, tandis qu'il est physiquement prisonnier d'un lieu provisoire. Le transmigrant précaire est sédentaire, mais sans attache.

Pour illustrer cette catégorie particulière de transmigrant précaire nous allons décrypter le documentaire Au bord des villes (2005), d'Aysun Bademsoy, scénariste allemande d'origine turque, qui est consacré à d'anciens travailleurs immigrés turcs retournés en Turquie après des décennies passées en Allemagne. Ces personnes vivent à Mesrin dans une ville portuaire du sud de la Turquie, et habitent de grandes cités dont la structure et l'aspect sont inhabituels pour ce pays : de gros blocs d'immeubles d'habitation entourant une sorte de parc. Au milieu de ce parc : de grandes piscines, des lieux de convivialité, des restaurants et des bars. Le film brosse le portrait de six familles qui parlent de leur expérience migratoire et de leur vie actuelle. En complément des interviews, le film présente l'architecture extérieure et intérieure qui constitue le cadre de vie de ces migrants "revenus au pays".

\section{Au bord des villes, des portraits marqués par l'ambivalence}

Scène $1:$ le jeune homme au bord de la mer. La présentation de ce jeune homme assis dans son auto, regardant la mer, souligne l'ambivalence intérieure du personnage dans sa relation à l'Allemagne et à la Turquie. Il déclare : "Si j'étais resté en Allemagne, ma vie se serait déroulée de manière plus ordonnée", ce qui est contredit par l'affirmation qui suit : "Si je retournais en Allemagne, je ne pourrais plus y vivre." "Maintenant, je suis heureux" contraste avec le deuil très vivant et profond de la perte de son père ; le personnage commente ainsi son expérience de la solitude 
durant la première phase du retour : "Il y avait le vide, la solitude." La vie en Turquie est perçue comme provisoire: "Aujourd'hui encore, jattends que cela change. Qu'est-ce que je vais devenir ? [...] Qu'est-ce qui va se passer? Tout est incertain.” Le mouvement de la caméra sur la mer accentue l'impression d'une forte nostalgie et de la perte de la patrie.

Scène 2 : le retour dans un pays étranger. Le retour en Turquie est ressenti par la jeune femme interviewée comme positif et négatif à la fois. Son comportement est aussi ambivalent : "Nous étions revenus pour toujours, c'était clair. Ce n'était bien sûr pas une bonne chose. Mais mes parents avaient raison eux aussi." La nostalgie de l'Allemagne est le motif central de cette scène. C'est l'Allemagne qui est ressentie comme la patrie perdue. C'est particulièrement net quand l'enfance est évoquée à travers le souvenir de mets qui n'existent qu'en Allemagne. La Turquie est un pays étranger, et elle le demeure.

Scène 3 : "une vie gâchée". Le souvenir particulièrement fort de l'expérience de la migration et des émotions qui l'accompagnent prime sur la perception de l'existence individuelle. La personne présentée, qui vit en Turquie, est cependant liée à l'Allemagne à travers son psychisme et ses émotions. Ce n'est pas le va-et-vient, la mobilité physique, qui rend impossible une "arrivée" en Turquie, comme la recherche sur l'immigration l'a jusqu'à présent supposé, mais c'est le contrecoup émotionnel de la mobilité. L'homme en question pleure sa vie manquée. L'expérience de la migration est comparée à la mort. "L'homme s'habitue à beaucoup de choses, en particulier à la mort. La mort. La mort." La migration a causé une rupture durable avec ceux qui sont restés en Turquie: "Ici, les gens ne se doutaient de rien. Ils n'avaient aucune idée de la façon dont on devait s'éreinter." L'Allemagne, c'est le passé, mais les émotions et les souvenirs rendent l'enracinement en Allemagne si fort qu'une véritable "arrivée" en Turquie a été impossible. Le long plan fixe sur le téléviseur, à la fin de la scène, suggère l'anonymat et souligne la solitude du personnage.

Scène 4 : un univers de vacances.Les migrants n'ont pas d'ancrage physique non plus. Cela se remarque par la représentation détaillée du lieu où se déroule leur nouvelle vie. Les longues séquences montrant des immeubles résidentiels et une piscine évoquent un univers de vacances. Le territoire résidentiel évoque une situation provisoire, un lieu que l'on quitte, en principe, "à la fin des vacances". C'est en même temps un lieu qui protège ses habitants de son environnement. Il est fermé, sécurisé, privé. 


\section{L'existence des transmigrants manque de reconnaissance politique}

Au bord des villes montre la vie "entre deux" nations, avec son caractère provisoire. Les transmigrants n'ont pas franchi à plusieurs reprises les frontières, ni physiquement, ni psychiquement, et sont plutôt bien sédentarisés. Mais le lieu où ils vivent n'est pas pour autant un lieu où ils seraient véritablement "arrivés", il s'agit plutôt d'un environnement provisoire qui reflète leur manque d'ancrage intérieur. Leur monde psychique est marqué par l'ambivalence. Néanmoins, ils ne vivent pas - comme le transmigrant traditionnel - dans un espace social transnational. Les relations sociales qu'ils entretenaient en Allemagne sont perdues, leur monde intérieur se confond avec un espace du souvenir qui ne tient pas compte des frontières, et se traduit par un déchirement entre les deux mondes. Leur "arrivée" émotionnelle et psychique en Turquie est entravée. Par son film, Aysun Bademsoy rétablit l'équilibre par rapport à la représentation jusqu'alors essentiellement positive du transmigrant dans les médias, dans la politique et dans la recherche. Il s'agit ici surtout de reconnaître politiquement la place des transmigrants dans l'histoire allemande de l'immigration.

Accorder la double nationalité aux anciens migrants et à leurs enfants et petitsenfants serait un geste positif dans ce sens. L'existence et les problèmes des transmigrants précaires doivent être pris en compte et faire l'objet de débats. Pour que le projet de réforme de l'intégration, appelé Reformprojekt Integration, réussisse, il faut tenir compte, à la suite d'Aysum Bademsoy, des conséquences négatives et douloureuses de l'émigration, du retour au pays et de la transmigration.

Traduction de Françoise Toraille

1. Portes, Alejandro, Guarnizo, Luis E. et Landolt, Patricia (1999), "The study of transnationalism : pitfalls and promise of an emergent research field", Ethnic and Racial Studies, 22, 2, p. 217-237.

2. Pries, Ludger (2000) : Transnationalisierung der Migrationsforschung und Entnationalisierung der Migrationspolitik. Das Entstehen transnationaler Sozialräume durch Arbeitswanderung am Beispiel Mexiko USA. In : IMIS-Beiträge,

15/2000, S. 55-77, p 59.

3. Pries, Ludger (2007: 34) 